\title{
Studien zur Geographie Palästinas bes. im Neuen Testament.
}

Von Jullus Boehmer in Raben bei Wiesenbarg (Bez. Potsdam).

\author{
I. Samaria Stadt oder Landschaft? \\ $\mathrm{Zu}$ Apg 8, 5 .
}

Samaria, das ursprünglich einen Berg bezeichnete (vgl. I Kön 16, 24) und seit Onri Stadtname war, ist bald Name des ganzen von der so benannten Residenz aus regierten Reichs und nach dessen Untergang der zwischen Judäa und Galiläa eingebetteten Landschaft geworden. Besonders seitdem die öfter zerstörte und immer wieder neu aufgebaute Stadt durch Herodes, dem Augustus sie zum Geschenk gemacht hatte, als Sebaste (heute: Sebásțje, nicht wie man fast überall lesen kann: Sebasțije - die heutigen Landesbewohner betonen das Wort ausnahmlos als Paroxytonon) in neuer Herrlichkeit wieder erstanden war, galt der Name Samaria ausschließlich der Landschaft.

Eine einzige Ausnahme scheint im Neuen Testament Apg 8, 5 zu bieten. Denn hier ist nach den besten Textzeugen Sin. Alex. Vat. zu


die Stadt Samaria", den Genitiv also als gen. appos. genommen. Neuer-

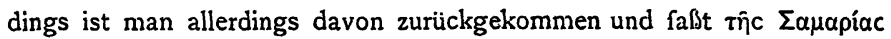
als Landschaftsbezeichnung, um zu übersetzen: „in die Stadt Samarias“, worunter dann die Hauptstadt Samarias zu verstehen sein solle. Nun haben andere Textzeugen wie Ephr. Bez. und ihnen nach der receptus


neuerdings Wilke-Grimm im neutestamentlichen Wörterbuch als "die richtigere Lesart" erklärt. Dabei geht er allerdings von der eigentümlichen Voraussetzung aus, daß. die Lesung mit tìv den gen. appos. $z u$ fassen gebiete (,in die Stadt Samaria"), während die ohne tìv den gen. partit. anzunehmen heische (,in eine Stadt von Samaria"). Im ersten Fall wäre also Samaria als Stadt, im zweiten als Landschaft zu nehmen. Aber warum soll nicht, um bei dem herrschenden Sprachgebrauch zu bleiben, auch bei jener Lesart Samaria als Landschaftsname, der Genitiv also auch dort als gen. partit. genommen werden? Dann kann und muß auch der Artikel $\tau \dot{\nu} \nu$ stehen bleiben, den zu beseitigen gegen die drei 
besten Textzeugen überhaupt seine Schwvierigkeiten, fast unüberwindliche, haben würde.

Aber was ist nun unter der Stadt Samarias zu verstehen? Man sagt: die Hauptstadt. Natürlich, aber welches ist die Hauptstadt Samarias zur Zeit des Neuen Testaments? H. Holtzmann im Kommentar nimmt die Frage auf die leichte Achsel und entscheidet (S. 63): da Samaria laut V. 9. I4 das Land bezeichnet, so „wird hier Sebaste, das frühere Samaria, als Hauptstadt des Landes gemeint sein; fehlt der Artikel, so ist eine beliebige andere Stadt gemeint". Danit aber hat sich der Erklärer die Entscheidung doch ein bischen zu leicht gemacht. Hingegen in J. IVeib' Schriften des Neuen Testaments hat Knopf, der sich mit Bestimmtheit für die erste Lesart ausspricht, folgerichtig dann auch Samaria als Sebaste genommen. Das liegt sicherlich am nächsten, solange man in der ganzen Frage auf rein geographischem Boden verharrt. Allein nun ist Samariter bekanntlich in erster Linie, mindestens für das neutestamentliche Zeitalter, kein geographischer, sondern ein religionsgeschichtlicher Begriff. Das liegt nicht nur der Darstellung in Apg 8, die in V. 25 ausmündet (,viele Dörfer der Samariter"), zugrunde, sondern ist auch in den Evangelien-Stellen offenbar., vgl. Matth I0, 5. Luk 9, 52. I0, 33. I7, 16. Joh 4, 9. 39. 40. 8, 48. Selten blickt hier die geographische Grundlage durch, wie Luk 9, 52: an den übrigen Stellen ist mit "Samariter" ganz deutlich ein Religionsangehöriger bezeichnet, der zu Juden 'oder Heiden oder zu beiden der verschiedenen Religion wegen in Gegensatz gestellt wird. Am deutlichsten ist das vielleicht Joh 4. Dann aber kann der Name Samaria kaum anders gemeint sein. Er findet sich an folgenden neutestamentlichen Stellen: Luk I7, I I. Joh 4, 4 . 5. 7. Apg I, 8. 8, 1. 5.9. 14. 9, 31. 15, 3. Auch hier ist in Luk I7, II die geographische Grundlage nicht $2 \mathrm{u}$ verkennen, wiewohl im folgenden (V. 16) die religionsgeschichtliche Orientierung doch wieder durchbricht. An anderen Stellen aber herrscht diese vor: zu Joh 4 wird weiter unten (S. 4 ff.) das Nötige gesagt werden. In Apg I, 8 steht „Judäa, Samaria und das Ende der Erde" neben einander als religionsgeschichtliche Bezeichnung der Juden, Samariter und Heiden. Nur Apg 15, 3 ist Samaria rein geographischer Name und ähnlich Apg 9, 31, wo allerdings, indem "Samaria" hinter "Judäa und Galiläa" steht, das geographische Prinzip, das Samaria zwischen Judäa und Galiläa erfordert hätte, von dem religionsgeschichtlichen, das die Samariter den Juden nachzuordnen gebot, durchkreuzt wird.

So ist auch der Sinn der Erzählung Apg 8, I-25 dieser, daß und 
wie das Evangelium zur Religionsgemeinde der Samariter (nicht in das Land Samaria) gelangtc. Der Abschnitt ist daher in Parallele zu der Darstellung vom Übergang dos Evangeliums in die Heidenwelt zu setzen: anders nuüßtc irgendwo auch eine cntsprechende Erzählung von Galiläa stehen. Die findet sich aber nicht: so oft Galiläa oder Galiläer in der Apostelgeschichte vorkonmt, ist es rein geographisch und ohne Beziehung auf das Missionswerk gemeint, z. B. außer 9, 31 noch 10, 37. 13, 31. Daraus versteht sich nun auch, daß im ganzen Abschnitt nur von Samaria im allgemeinen die Rede ist, und keine Örtlichkeit genannt wird. Zivar wird V. 5 "die Stadt" erwähnt und V. 9 wiederholt, aber ihren Namen zu nennen wird nicht für die Mühe wert gehalten. Am deutlichsten wird das V. 14, wo, nachdem vorher nur von "der Stadt" erzählt worden ist, es jetzt auf einmal heißt: „Samaria" habe das Wort Gottes angenommen, und dann fortgefahren wird: Petrus und Johannes seien „zu ihnen“ entsandt worden. Man hat hier erst recht den Eindruck, daß, weil nicht das zu erwartende "dorthin" gebraucht wird, es vor allem auf die Personen als Glieder der samaritischen Religionsgemeinde ankomme. Nimmt man dazu den so allgemeinen und blassen Abschluß V. 25, daß „die Apostel nach Jerusalem zurückkehrten und (doch wohl auf dieser Rückkehr) vielen Dörfern der Samariter das Evangelium brachten", so wird wohl völlig klar, eben aus dem Mangel aller geographischen Genauigkeit (wie anders Kap. 13 ff. die Angabe jeder Reisestation!), daß die Samariter hier nur als sozusagen halbe Juden in Betracht kommen und die Samaritermission den Übergang zur Heidenmission bildet, eher noch auf gleicher Stufe mit der Judenmission steht.

Ist dem aber so, dann kann unter der "Stadt der Samariter" nur das Zentrum der samaritischen Religionsgemeinde, also Sichem-Nablus verstanden sein, umsomehr, als Samaria-Sebaste schon seit Alexander dem Großen hellenisiert war. Die Makkabäer haben sie bekämpft, Hyrkanus' I Söhne sie erobert, weil sie hellenistische, also heidnische, nicht weil sie samaritische Ketzerstadt war. Dazu kommt, daß bald nach Herodes' Tode Sebaste überhaupt zuriickging und sehr schnell von Sichem-Neapolis überflügelt wurde, so $\mathrm{da} ß$ es für den Verfasser der Apostelgeschichte schiverlich eine derartige Rolle mehr spielte, um, noch dazu an Stelle von Sichem, überhaupt auf Erwähnung rechnen zu können.

\section{Zu Ev. Joh. 4 (Sichar-Sichem u. a.)}

I. Es bedarf keiner Worte, daß die Joh 4, 5 bezeichnete "Stadt Samarias" mit dem Namen "Sichar" bezeichnet wird. Die Versuche, die 
man früher gemacht hat, statt Sichar vielmehr Sichem zu lesen, oft genug mit Berufung auf Eusebius-Hieronymus' Onomastikon (was sich z. T. bis in die neueren Kommentare hinein fortschleppt) sind als gescheitert zu betrachten und endgültig aufzugeben. Denn Eusebius' Onom.

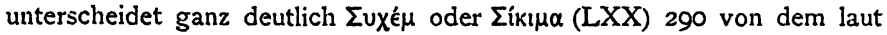
297 östlich von Neapolis-Sichem gelegenen Euxúp. Auch kennt der Talmud an mehreren Stellen (Baba Kamma 82, Menachoth 64) ein סוכר = Suchar, dessen Lage allerdings nicht näher bestimmbar ist. Immerhin gibt uns das einen Anhalt dafür, wie der Verfasser des Evangeliums auf die Lesung 乏uxá $\rho$ kam. Denn die Frage, ob die so bezeichnete Ortschaft mit dem gegenwärtig rund $I \mathrm{~km}$ nördlich vom Jakobsbrunnen gelegenen Dorf 'Askar oder Ischar zusammenfalle, muß von der SicharSichem-Frage völlig unabhängig gehalten werden. Es ist nämlich durchaus möglich, daß der Name 'Askar, wenn er wirklich mit Sichar zusammenhängt oder aus Sichar entstanden ist, seiner Zeit dem betreffenden Ort, sei es, daß er schon bestand oder erst gegründet wurde, auf Grund von Joh 4, 5 erst beigelegt ward. Ähnliches mag von dem Namen „Jakobsbrunnen" und seinem gegenwärtigen Träger gelten.

Schon hiernach sind die älteren Versuche, die Identität von Sichar und Sichem darzutun, sei es, daß man Sichar als andere (härtere) Aussprache von Sichem nahm oder als absichtliche Annäherung an (= Lüge laut Hab 3, IS) oder (trunken, laut Jes 28, I) faljte, keineswegs a limine von der Hand zu weisen. Es liegt ihnen, so gekünstelt sie mitunter scheinen mögen, ebenso wie den Bestrebungen, Sichem für Sichar einzusetzen, überhaupt die durchaus richtige und gesunde Empfindung zugrunde, dab man im Gesamttenor von Joh 4 Sichem, nicht Sichar zu erwarten hätte.

Das ganze Kapitel nämlich zeigt, wie das Evangelium überhaupt, trotz der scheinbar so bestimmten und präzisen Ortsangaben, gerade eben auf diesem Gebiet auch wieder eine merkıürdige Sorglosigkeit und daraus folgende Unklarheit. Es fällt schon auf, daß es nach V. 4 ,er mubte durch Samaria reisen" gleich V. 5 weiter heißt: ;er kommt nun in eine Stadt Samarias namens Sichar", noch mehr aber V. 8: „da kommt ein Weib aus Samaria“. Ist schon in V. 5 die Wiederholung Samarias im Zusammenhang merkwürdig weil überflüssig, so fällt in V. 7 erst recht die Betitelung "Weib aus Samaria" auf. Denn, ist hier mit Samaria die Landschaft gemeint, dann ist ihre Erwähnung wieder überflüssig, um nicht zu sagen: seltsam (denn naturgemäls wohnten in Samaria samaritische Frauen). Oder: ist an die Stadt Samaria ge- 
dacht, dann ergäbe sich der Widersinn, daß die Frau, da der Jakobsbrunnen etwa $2 \mathrm{~km}$ östlich von Sichem und dieses über zwci Stunden südöstlich von der Stadt Samaria liegt, rund drei Stunden marschiert wärc, un IVasser $z$ u holen. Selbstverständlich ist hier beide Male voraus-


von $E_{K} \kappa \tau \lambda$. mit ruví schr hart und ganz ungriechisch wäre, im folgen-

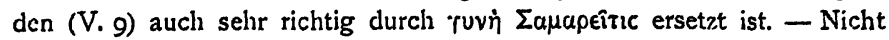
minder deutlich ist V. 5 die Ausdrucksweise: $\epsilon i c \pi o ́ \lambda$ เv. Die neueren Übersetzer driicken sich um die hier vorliegende Schwierigkeit herum, indem sie dies mit „an eine Stadt" „zu einer Stadt" wiedergeben, wofür in Griechischen bekanntlich andere Präpositionen zur Verfügung gestanden hätten: einzig Weizsäcker übersetzt herzhaft mit Luther ,in eine Stadt's. Warụm sich jene an der nächstliegenden und einzig richtigen Übersetzung vorbeidrücken, zeigt V. 8, wo es heißt, die Jünger seien „in "die Stadt" gegangen, um Speise zu kaufen, während Jesus am Jakobsbrunnen $\mathrm{sab}$. So konnte man sich allerdings eigentlich nicht mehr ausdrücken, wenn Jesus mit seinen Jüngern laut. V. 5 bereits in die Stadt gekommen war. Des ganzen Rätsels Lösung ist einfach die Sorglosigkeit des Evangelisten in allen das Äußere betreffenden Angaben. Aber auf Kosten dieser ist derselbe um so genauer und deutlicher in der $\mathrm{Be}$ zeichnung und Hervorhebung dessen, worauf es ihm ankommt. Und das ist hier das Moment des Samaritischen. Daher in V.4.5 unmittelbar nebeneinander die zweimalige Hervorhebung von Samaria, daher das aus Samaria kommende Weib V. 7 (ob der Evangelist hier an die Landschaft oder an die Stadt dachte, darf man eigentlich nicht einmal fragen - es kam ihm nichts darauf an, wenn er überhaupt über die Lage der Stadt Samarias genauer orientiert war), daher V. 9 die zweimalige Nennung des samaritischen Weibes (mindestens das erste Mal

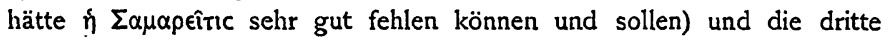
Erwähnung der Samariter im gleichen Verse. Jetzt endlich meint der Verfasser, nachdem er in wenigen Zeilen hintereinander sechsmal auf Samaria hingewiesen, deutlich genug gemacht zu haben, worauf es ihm ankomimt. Erst V. 39. 40 kehren dann die Samariter ziveimal wieder, und hier wird nun vollends deutlich, was V. 4-9. vorbereitet wurde und dem Ahnungsvermögen überlassen blieb, daß "Samaria“, „Samariter" dem Evangelisten religionsgeschichtliche, keine geographischen Begriffe sind: sonst hätte er wohl von den Leuten der Stadt wenigstens einmal als "Sichariten" oder ähnlich geredet.

Ist dem so, dann wird sich der Schluß schwerlich umgehen lassen, 
daß auch „die Stadt Samarias", die Stadt der Samariter, gleichviel ob sie Sichar oder wie sonst heißt, in religionsgeschichtlichem Sinne zu nehmen ist. Für die Religionsgeschichte aber ist Sichem-Neapolis „die Stadt der Samariter" Kat' 'Ěoxńv allzeit gewesen und noch heute. Wollte der Evangelist Jesum unter den Samaritern missionieren lassen, dann hätte er es recht ungeschickt angestellt, wenn er Sichar als Missionsgebiet erwählte und dies ein kleines Städtchen gewesen wäre - von Jesus selbst ganz zu geschweigen, der an einem dem Jakobsbrunnen so nahe gelegenen Zentrum wie Sichem vorübergegangen sein sollte, um jenen kleinen Ort (gleichviel ob es 'Askar war oder nicht) mit seinem mehrtägigen Besuch zu beehren. Missionstätigkeit Jesu in Samaria gehörte nach Sichem und nicht nach Sichar, ebenso wie Jesus in Kapernaum und niclit in 'Akbara, in Jerusalem und nicht in Mizpa auftrat.

So angesehen ist die Frage, was Sichar eigentlich ist, ziemlich irrelevant. Auch die Frage, wie diese Stadtbezeichnung in den Text geraten ist, darf auf sich beruhen. Vielleicht hat der Evangelist Sichem und Sichar verwechselt, vielleicht hat er aus irgend einem der oben angeführten oder aus einem anderen Grunde mit Absicht Sichar statt Sichem geschrieben. Vielleicht ist Sichar erst später in den Text eingesetzt worden, als man bemerklich machen wollte, daß Jesu Weg direkt nach Norden führte, am Jakobsbrunnen vorbei und über Sichar-'Askar nach der Jordan-Ebene und zum See Genezareth. In diesem Fall hätte man dann freilich übersehen, dals es eigentlich recht ungeschickt war, V. 8 die Jünger zum Ankauf von Lebensmitteln in die Stadt $z u$ schicken, die man doch später jedenfalls gemeinsam passieren mußte, und so die Jünger $3 \mathrm{~km}$ machen zu lassen, wo $\mathrm{I} \mathrm{km}$ ausgereicht hätte. Ebenso hätten dann die Samariter V. 40 sich sehr überflüssigerweise zu Jesus bemüht und ihn zu sich bemüht, wenn Jesus ohnehin auf seiner Weiterreise vom Jakobsbrunnen aus binnen einer Viertelstunde ihren Ort berühren mußte. Dagegen ist alles klar, wenn Sichem gemeint ist, da Jesus dann tatsächlich auf seinem Wege nach Norden einen Abstecher vom Jakobsbrunnen aus $2 \mathrm{~km}$ westlich und später ungefähr denselben Weg zurück zu machen gehabt hätte.

2. Gerade vom Jakobsbrunnen aus wird der Anblick der schon von weitem sichtbaren herzueilenden Samariter, die von Sichem her den Bergrücken herab zwischen den grünen Feldern herzukamen, besonders eindrucksvoll gewirkt haben. Da nach V. 35 noch vier Monate bis zur Ernte waren, so muß zur Zeit, als Jesus diese Worte sprach, Januar gewesen sein: die Ernte fallt in den Mai und hebt in nur besonders 
guinstigen Jahren schon im April an. Dem widersprechen auch nicht, wiewohl man das oft gesagt hat, Bemerkungen wie die V. 1. 2. 6. 7. Denn auch um die Jahresivende gestattet es die Temperatur in der Niederung des Jordans in der Nähe des Toten Meercs, wo Johannes der Täufer gewirkt hat, daß getauft wird. Zudem wäre wohl denkbar, daf, die Taufen im Monat Oktober eingestellt worden sind und Jesus doch erst im Dezcmber oder Januar aus Judäa aufbrach, weil Gerüchte wie die V. I erwähnten oft genug längere Zeit brauchen, bis sie an die richtige Adresse gelangen. Indes, wie gesagt, bedarf es nicht einmal dieser Annahme, da der subtropische Charakter der Gegend, wo die Taufstelle lag, das immerhin nur kurze Tauchbad im Jordan erlaubte, und zudem die Menschen in Jesu Zeitalter noch abgehärtet genug waren, um auch bei einer den Modernen empfindlich kühl erscheinenden Temperatur ins Wasser zu tauchen. Man muß, um dies verständlich zu finden, nur Zeuge einer in einer griechisch-orientalischen Kirche stattfindenden Taufe gewesen sein. Hier wird noch heutiges Tags der Täufling innerhalb seiner ersten Lebenswochen unbekleidet in das mit Brunnen- oder Cisternenwasser gefüllte Taufbecken völlig hineingetaucht: eine Prozedur, die in unseren Augen an Grausamkeit nichts zu wünschen übrig läbt. Als daher einmal ein europäischer Christ, der einer solchen Taufe als Pate beiwohnte, völlig überrascht von dem ihm ungewöhnlich erscheinenden Verfahren, unwillkürlich den Ausruf: ja mäskîn (=o du Armer) ausstieß, da wurde ihm von einem der umstehenden Eingeborenen die Antwort zuteil, daß immerhin die Schmerzempfindung des Täuflings längst nicht hinanreiche an die Schmerzen, die ein muhamedanischer Knabe bei der Operation der Beschneidung auszustehen habe.

Auch daß Jesus sich nach V. 6 auf den Brunnen setzte, hat in der Gegend von Sichem, selbst im Dezember und Januar, nichts Befremdliches, umsomehr, als es um die 6. Stunde, also gerade Mittagszeit war. Damit erledigt sich zugleich das Bedenken, als ob nicht nach V. 7 ein Weib damals Wasser geschöpft haben könnte: denn nur für die Mittagszeit ist es vorstellbar, daß eine einzelne Frau den Brunnen aufsuchte, während sonst in der Regel die Frauen gemeinsam ihren Wasserbedarf, noch dazu in so weiter Entfernung von der Stadt, befriedigen. Freilich auch selbst diese Regel wird nicht unbedingt innegehalten. Vielmehr sieht man öfter noch heutzutage einzelne Frauen zum Brunnen nach Wasser gehen. Das ist an unserer Stelle um so natürlicher, als die Frau, wenn sie auch nach Sichem gehörte, sicher nicht eigens aus der Stadt kam, um Wasser zu holen (es gab bei Sichem Quellen genug), sondern 
vom Acker oder von der Weide in der Umgegend des Brunnens hinging, um für ihre Feldarbeit oder für das Vieh oder zu welchem Zweck es sonst sein mochte, Wasser zu schöpfen.

Aber all derartige Reflexionen werden dem Evangelisten völlig fern gelegen haben: ihm kam es nur darauf an, die Begegnung Jesu mit der Samariterin am Brunnen und daraus den Gegenstand ilhres Gesprächs zu motivieren, während er in den das Äußere betreffenden Angaben, so auch wegen der Möglichkeit oder Wahrscheinlichkeit des Brunnengangs der Frau in diesem Zusammenhang, vollkommen sorglos war.

\section{Tyropöon.}

In der vielverhandelten Beschreibung Jerusalems, die Josephus in seiner "Geschichte des jüdischen Krieges" V 4 bietet, heißt es $\$$ I am Schluß: „Das Tal, das den Hügel der oberen Stadt von dem der unteren

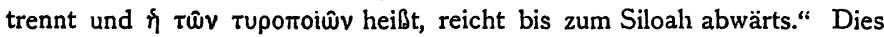
ist die einzige Stelle, in der das von Norden nach Süden hinabgehende Tal, das den Westhügel vom Ostflügel schied, mit Namen genannt wird. Der Name ist seltsam und gibt viel zu denken.

Die landläufige und so ziemlich allgemein angenommene Erklärung versteht den Namen, wozu freilich der griechische Name herausfordert, als „Käsemacher-Tal“. Nun haben wir freilich keinerlei Nachrichten darüber, wie das $\mathrm{Tal}$ zu einem derartigen Namen gekommen sein sollte. Vermuten darf man ja allerlei. Man muß schon in alte Zeiten zurückgehen, wo Israel noch wesentlich ein Ackerbau und Viehzucht treibendes Volk war, wo auch die Hauptstadt Jerusalem selbst noch ein Landstädtchen mit beträchtlichem landwirtschaftlichem Betriebe war, um es begreiflich zu finden, daß eine Lokalität nach dem Gewerbe der Milchverarbeitung und Käsebereitung seinen Namen empfangen hätte. Allein gerade Jerusalem und seine Umgebung macht es bei seiner Bodenart etwas schwierig, daran zu glauben, daß es dort je Weideland in größerem Maßstabe gegeben hätte. Aber selbst wenn Käsebereitung, die doch bequemer und daher naheliegender Weise draußen, auf den Weiden, in den Fütten der Hirten hätte getrieben werden sollen, in der Stadt vorkam, ob sie je in so großem Umfang hier geschah, dab man eine ganze, noch dazu ziemlich lange Straße darnach benannte?

Bedenken, die nicht leicht zu überwinden sind. ${ }^{x}$ Aber nun hat man gemeint, eine Stütze für das „Käsemachertal“ im „Käsetor" zu finden.

I Um das Urteil zu erleichtern, sei erinnert, daß im heutigen Jerusalem zwar Rinder, Schafe und Ziegen im Stall gehalten werden, dagegen keinerlei Käsebereitung 
Nch 3,13 erwähnt cin soll nach I Sam 17, 29 "Käse“ heißen. Allein rist 1 Sam 17, 29, das schon LXX nicht mehr verstanden und daher unübersctzt ließen, ist tatsächlich noch immer unerklärbar, namentlich neben Eirklänung harrt. Neh 3, 13. abes kann gemäb 2, 13 nur siự gelesen werden, so daß cin "Misttor" herauskommt (LXX haben hier beide Male

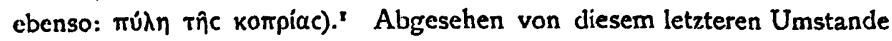
stehen wir hier vor lauter Unsicherheiten, so da $\$$ wir nach Lage der Dinge gut tun, nicht auch diesen einen sicheren Umstand noch fahren zu lassen.

Aber wie ist der Name Tupotooŵv zu verstehen? Selbstverständlich hat ihn Josephus nicht aus der Luft gegriffen. Allein an sich wäre es schon merkwürdig, wenn dieses $\mathrm{Tal}$, das doch von Alters bestand, in der Landessprache keinen Namen gehabt hätte, sondern gleichsam erst auf das hellenistische Zeitalter hätte warten müssen, um benannt zu iverden. So sind denn auch beispielsweise die im selben Kapitel angeführten topographischen Bezeichnungen für Jerusalem allesamt hebräischaramäischen Sprachcharakters: Silva, Bethso, Ophla, Gennath, Kedron (von Bezetha, das u. E. auch hierher gehört, sehen wir ab, weil sein Verständnis ein gespaltenes ist, s. S. 226 A.), während die vorkommenden griechisch-römischen Bezeichnungen, nämlich: Akra, Hippikos, Xystos, Psephinos, Antonia, durchweg erst der (sogar spät-)hellenistischen Periode ihr Dasein verdanken. So legt sich die Annahme nahe, daß Tupomolŵv nichts als die Gräzisierung eines semitischen Äquivalents ist.

Aber welches ist dieses? - Von dem biblisch-hebräischen טרן

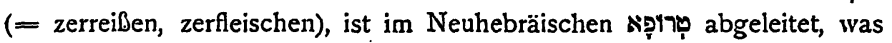
„Bach (oder Fluß) mit reißender Strömung" bedeutet" (vgl. Levy Neuhebr. Wbuch II 197a). Es ist nicht zu bezweifeln, dab in dem in Rede

getrieben wird. Diese findet in mehreren $D$ örfern der Umgegend, vor allem aber in Nablus statt. [Zur Zeit, nebenbei, gelten die Trappisten in Lātrūn dafür, daß sie den besten, aber auch teuersten Käse herstellen.] Dörfer, die Nilch und Käse nach Jerusalem liefern, sind besonders: eț-Ṭūr, Mālhạ, Liftā.

I Buhl, Geographie S. 132, will 'Tyropoion auf einen Euphemismus zurückführen, indem ein ursprüugliches ašfot = Mist in šafot-Käse umgewandelt worden, und so aus Misttal Kãsemachertal entstanden sei. Buhl kann sich dafür auf Halévy berufen. Allein abgesehen davon, daß die Bedeutung šafot-Käse, wie gesagt, durchaus nicht feststeht (vgl. d. Lex.), und ferner "Käsemacher" nicht dasselbe wie Käse ist, will es wenig einleuchten, dals das ganze $\mathrm{Tal}$ nach dem Mist, der an seinem Ausgang dureh das Misttor hinausgeschafft wurde, seincn Namen getragen hätte.

2 Vgl. auch den (seltenen) Namen 1rọ̄, den der bekannte Tannaite-Gegner von


Strack, Einl. i. d. Talmud S. 89 u. 90 Anm.). 
stehenden Tal in alten Zeiten, als es noch wirklich ein Tal, ein Einschnitt zwischen zwivei Höhen (eine páparž nach Josephus, d. i. Schlucht, Kluft) war, zur Regenzeit ein Bach lief, wie noch heutigestags nach jedem Regenguß die Straßen der Innenstadt Jerusalem im Wasser, das eiligst dahinrinnt, schwimmen. Das Gefälle dieses Bachs nämlich, der von seiner Entstehung im Norden des Damaskus-Tores bis zur Einmündung ins Kidrontal etwa $2 \mathrm{~km}$ durcheilte, muß außerordentlich stark gewesen sein, da von der Gegend des „Aschenhügels“ ( $8 \mathrm{I} 4 \mathrm{~m}$ hoch) bis zum Kidrontal (an der Einmündungsstelle $620 \mathrm{~m}$ hoch) fast $200 \mathrm{~m}$ Höhendifferenz vorliegt, also auf $\mathrm{I} \mathrm{km}$ im Durchschnitt roo $\mathrm{m}$ Gefälle. Das ist ein Gefälle, wie es keins der Täler in und um Jerusalem aufzuweisen hatte. Das macht aber, weil die beiden anderen Haupttäler, das Kidronwie das Hinnomtal durch den Bogen, den sie an der Nordost- oder Südwestecke Jerusalems beschreiben, ihren Lauf verlängern, während das Tyropöon von Norden nach Süden in ziemlich gerader Richtung sich forterstreckte und die erste nennenswerte Biegung erst kurz vor der Einmündung ins Kidrontal machte. Leider kann man einen Eindruck davon heutzutage nicht mehr gewinnen, weil gerade das Tyropöon genannte Tal mit Schuttmassen von 20-30 m bedeckt ist und der Proze $\$ seiner Zuschüttung überhaupt schon ziemlich früh begonnen hat. Aber unter diesen Umständen begreift es sich wohl, warum man gerade dieses $\mathrm{Tal}$ vor anderen mit dem Namen טרופא auszeichnete, der an sich ja. auch dem Kidron- oder Hinnomtal hätte zufallen können. Im Alten Testament gilt als das $\mathrm{Tal}$ im besonderen $\mathrm{Sinn}$, in dem alle anderen sich vereinigen, das Kidrontal, das daher auch kurzweg הָ heißt (Neh. 2, 15. 2 Chron 33, 14), also das „Bachtal“. Dagegen das Hinnomtal heißt immer s'd, und zwar von Haus aus auch bloß $($ Jer 2, 23). Demnach hätte man die drei wichtigsten Täler Jerusalems ursprünglich nicht mit einem Eigennamen bezeichnet, sondern nach ihrer Sonderart unterschieden: das Südtal führte den allgemeinsten Namen העי = „das Tal“, das Osttal bestimmter הנחל „das Bachtal“, und das Westtal der ältesten Stadt (etwa) das Gielbachtal“, also am genausten gekennzeichnet.

Es kann nicht auffallen, dab gerade dieser letztere Name sich erhielt, während die anderen beiden Talnamen bald durch Eigennamen ausgezeichnet wurden. Denn sie wurden für die Stadt schon aus Gründen der Verteidigung wichtig und-zwangen immer wieder das Augenmerk der Bewolner auf sich. Dagegen das Westtal trat in diesem Sinne mehr und mehr an Bedeutung zurück, als auch der Westhügel bebaut wurde und sich zu einem Stadtteile auswuchs. Die Mauer der Stadt 
umschloß jedenfalls schon in dcr Königszeit ein Stück des Westhügels, und damit war das "Tyropöon" gleichsam außer Kurs gesetzt. Es behielt aber seinen alten Namen bis auf den heutigen Tag.

Donn, so scheint es, der Name מרומא entspricht dem Sinne nach ziemlich genau der heutigen Bezeichnung: el Wād - das Tal kat' 'Eoxív. Das Einzigartige diescs Tals ist hier ebcnfalls, wenn auch in anderer Weise, zum Ausdruck gebracht, wie in טרופא. Die Bezeichnung el-Wād ist um so auffälliger, als heute, im Unterschied von anderen Tälcrn, dieses Tal längst nichts Charakteristisches mehr hat; im Gegenteil sogar kaum mehr unter seinesgleichen als "Tal" erkennbar ist, von einem "reißenden Bach" ganz zu geschweigen. Der Name el-Wād erklärt sich so in der Tat am besten als die arabische Úbersetzung oder auch nur Fortsetzung seines hebräisch-aramäischen Vorgängers טרופא.

Vielleicht aber darf man hier noch einen Schritt weiter gehen. Man könnte versucht sein, immer noch die Endung wv oder mindestens das $v$ am Schluß in tuporoıwv auffällig $z u$ finden. Auch darüber noch ein Wort. Man weiß, daß etwa in der Mitte der heutigen Stadt und zugleich in der Mitte seines Laufs das Tyropöon vom Westen her ein Seitental aufnimmt, das (vom Jafa-Tor her) gleichsfalls in starkem Gefälle und nach sehr kurzer Strecke in das Tyropöon einläuft; man erkennt dies Seitental noch jetzt deutlich, wenn man durch die Davidstrale zur Stadt hinabgeht. Hier muß vor Zeiten ein anderes טרו sich ergossen haben; so daß man von der Vereinigungsstelle beider an von טา, aramäisch zu reden Anlab hatte. Dieser Name mag darin auf das nord-südliche Haupttal nach und nach übertragen worden sein und das $v$ am Ende von tupotouwv zur Genüge verständlich machen.

Man braucht aber dies Seitental nicht unbedingt heranzuziehen. Man känn sich vielmehr: auch daran genügen lassen, in dem i am Schluß eine auch sonst nicht seltene Erweiterung, ein Afformativ zu erkennen, s. Kautzsch hebr. Gramm. $\$ 85$ u. Auch so würde sich eine

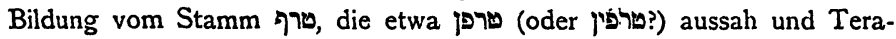
phān (Teraphjān) oder Terap̣hōn (Teraphjōn?) gesprochen wurde, zur. Genüge erklären und Tyropöon dann ganz deutlich, Buchstabe für Buchstabe, mit diesem hebräischen Äquivalent übereinstimmen.

Doch ob so oder so: jedenfalls sollte man vom „Käsemacher-Tal“ ablassen und erkennen, daß Josephus auch hier seinen Lesern zu lieb gräzisiert und uns in verhüllter Gestalt dennoch erkennbar genug den alten

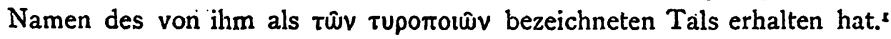

I Kaum bedarf es überhaupt der Erinnerung, daß auf Josephus' sprachliche und 


\section{Magadan.}

Im Anschluß an die Speisung der Viertausend berichtet Matthäus (und nur dieser) 15,39, daß Jesus in ein Fahrzeug gestiegen und in das Gebiet von Magadan gelangt sej. Diese geographische Bezeichnung kommt literarisch einzig an dieser Stelle vor und ist daher von jeher eine rechte crux interpretum gewesen. Den nächstliegenden Ausweg haben die Bearbeiter alter Handschriften gefunden und mit. Geschick "Magdala" an die Stelle gesetzt. Ob sie damit vielleicht instinktiv oder bewuBt das Richtige getroffen haben, darauf werden wir vielleicht noch zurückkommen müssen. Unsere ältesten Zeugen jedenfälls kennen nur Magadan, und sämtliche Erklärer bis auf die jüngsten sind sich darin einig, daß die so bezeichnete Ortschaft unbekannt sei, folgen zum Teil auch ihren Vorgängern alter Zeit, indem sie Magdala einsetzen.

Vielleicht aber lohnt es doch, nach einem Ausweg aus dieser schwierigen Lage zu suchen, gleichviel wo er sich bieten mag. Und er scheint nicht einmal so weit abzuliegen. $\mathrm{Zu}$ dem vorauszusetzenden palästinischen מגדן legtt sich ohne weiteres das altsteamentliche, nicht ganz unbekannte מֶּ מִּ als Ausgangspunkt dar. Es findet sich Deut 33, 13-16 im Joseph-Segen, ferner (in pluralischer Form) HL 4, I3. I6. 7, 14 im Sinn „edle Gabe“ sc. der Natur, also „reichliche köstliche Frucht". Das Äquivalent ist auch dem Neuhebräischen wie dem Syrischen

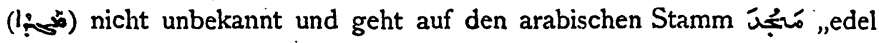

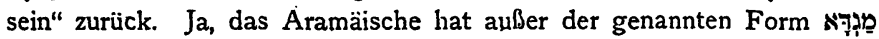

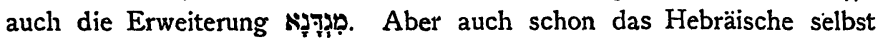
kennt eine entsprechende Erweiterungsform, nur daß sie uns (wohl zu-

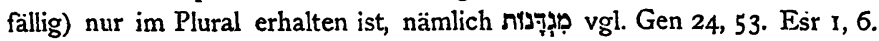
2 Chr 21, 3. 32, 23. Die Erweiterung des Stammes 7 zu den Substantiven mit angehängtem $\boldsymbol{l}$ ist ja nun nichts Singuläres. Kautzsch' Grammatik $\$ 85 \mathrm{u}$ stellt eine Fülle derartiger Nomina zusammen. Demnach hätten wir in Magadan eine Ortschaft- oder Landschaftbezeichnung zu erkennen, die eine besondere Fruchtbarkeit der Gegend andeutet.

Lälut man nun gelten, was nach dem Kontext bei Matthäus alles für sich hat, daß Magadan auf dem Westuier des Sees Gennezareth zu suchen ist, dann tritt als besonders fruchtbare Gegend wie von selbst die Ebene Gennesar, heute el-Ghuwēr genannt, vor uns hin. Bekanntlich hat Josephus sie ihrer Fruchtbarkeit wegen in einer fast dithyram-

etymologische Erörterungen wenig oder nichts zu geben ist, namentlich wenn es ihm galt, sich als guten "Griechen" zu zeigen. Man bedenke z. B., daß er im Bell. Jud. V 4, 2

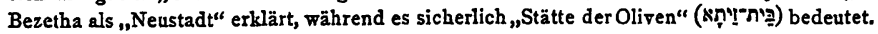


bischen Schilderung Bell. Jud. III 10, 8 verherrlicht. Ihr Name muß öfter gewechselt habcn: im Alten Testament heibt sie תרְִּ im Neuen Testa-

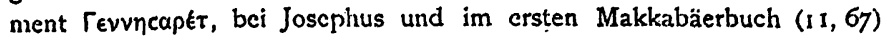
revuncúp, im Talmud sowohl wạ als als die allen diesen Formen zugrunde liegenden Stammformen sind (außer כנרת sind sie offenbar nur Variationen eincr einzigen Form), ist bisher nicht zu sagen. Man kann sogar vermuten, daß selbst die beiden Bildungen ת כנר und נגיסר nicht ganz außer etymologischem Zusammenhang untereinander stehen, indem für das $\Delta$ irgendwie epenthetische Natur (wie sonst für 7 und $ל$, seltener auch für 0 ), vielleicht infolge ausländischer Einflüsse, anzunehmen wäre. Doch dem sei, wie ihm wolle, selbst wenn גיסר nicht, was man öfter angenommen hat, mit j (Garten) zusammenhinge, so wird man zugeben müssen, daß die Bezeichnung "Magadan" im Sinne von "Landschaft mit reichlichen und köstlichen Früchten" ausgezeichnet auf das Gebiet Gennesar paßt, und da hier in der Gegend tatsächlich die Landungsstelle Jesu anzunehmen ist, so wäre das Mt 15, 39 vorliegende Rätsel bis auf weiteres leidlich gelöst. ${ }^{x}$

Vielleicht aber dürfen wir von hier aus noch einen Schritt vorwärts gehen. Von מגדן מst es offensichtlich nicht weit bis (מגדלה. Nun ist ja freilich das etymologische Verständnis von מגדל מגרל מגד als Mauerturm, Festungsturm, Festung nach allgemeinem Einverständnis so gesichert, daß daran rütteln $\mathrm{zu}$ wollen Unverstand wäre. Eine andere Frage ist aber doch, ob gerade der Ort Magdala, der an die Ebene Gennesar im Süden grenzt, durchaus in diesem Sinne zu verstehen ist. Denn auf die Übereinstimmung mit מגדל-אל, was Jos 19, 38 als feste Stadt in Naphtali erwähnt wird, ist kein Gewicht zu legen, da die Identität beider Städte nur vermutet wird. Noch mehr aber legt es die Lage unseres Magdala eben am Südrande von Gennesar nahe, sofern unsere Auffassung von Magadạn zutrifft, an die Ableitung vom Stamme מגach für diesen Namen zu denken. Nun gibt es bekanntlich im Hebräischen Weiterbildungen vom Verbalstamm nicht bloß mit i, sondern ebenso auch,

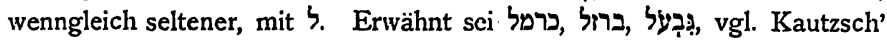
Grammatik $\$ 309,85 \mathrm{~s}$. Analog könnte von מגד in dem oben genannten

I Nach Wellhausen (Israelitische und jüdische Geschichte S. 220) ist Gennesar aus אì und Nesār = Galilaza zusammengesetzt. (Schon er erinnert, dẩ die Form Gennesaret im Anklang an Kinneret entstanden sei.) Dem gegenüber ist daran festzuhalten, dafs der Name der Landschaft ursprünglich, der des Sees abgeleitet ist, und dann bestimmt der erste Teil $\underline{\xi}=$ Garten lautet. Auch ist die Kombination von Nesār mit Galiläa unhaltbar und scheint auf ein Mißverständnis zurückzugehen, welches Nesār mit Nazareth (tendenziös) zusammenbringt. Vgl. Buhl, Geographie des alten Palästina S. 113 f. 
Sinne מגדל abgeleitet sein und so die „Stadt mit den reichlichen und köstlichen Früchten“ oder besser die „Stadt, die zur Landschaft mit den reichlichen und köstlichen Früchten (in irgend einem Sinne) gehört", meinen. $\mathrm{Da} b$ der Name, der ursprünglich so gedacht war und nach diesem seinem ursprünglichen Sinn vielleicht verborgen geblieben wäre, wenn nicht der. Evangelist Matthäus mit seiner seltsamen Form uns diesen Weg gewiesen hätte, später dann mit „Turm", „Festung“ zusammengebracht wurde, ist nicht zu verwundern. Anderseits aber, falls der Talmudz mit seiner Nachricht Recht hat, daß es in dieser Gegend mehrere Orte mit dem Namen Magdala gegeben habe, so ließe das auch darauf schließen, $\mathrm{da} \mathfrak{b}$ die fruchtbare Ebene Gennesar gerade es gewesen ist, die diesen Ortsnamen um des eben festgestellten Sinns willen $z u$ bevorzugen aufforderte.

Von hier aus ist vielleicht sogar die Frage, ob nicht der Name der kanaanäischen Stadt Megiddo gleichfalls im Zusammenhang mit מגד stehe, nicht a limine abzuweisen. $\mathrm{Dab}$ die gegenwärtige Form מִִּ̣ auf den Stamm גarückweise, mag sein. $D a ß$ es so sein müsse und daß es ursprünglich so gewesen sei, läßt sich keineswegs sagen. Die assyrischen Formen magādu und magidu, die Amarna-Formen makida (neben magidda) zeigen, daß den Fremdlingen die Aspiration des $d$ nicht wesentlich erschien. Ohne Frage hätte ein Name, der fruchtbare Gegend andeutete, für eine Stadt in der Kison-Ebene recht wohl in Betracht kommen können.

Endlich liest die syrische Bibel den Namen Magadan als o: gleichsam als wolle sie damit den Zusammenhang zwischen Magadan-Magdal(a) auf der einen Seite und Megid(d)o auf der anderen Seite erhärten helfen und an ihrem Teile zeigen, daß Magadan, Magdala, Megiddo im Grunde genommen Variationen einer und derselben Grundform sind. Vor allem aber ist nach der syrischen Form eins klar, daß wenn sie Magādu, also ganz wie im Assyrischen, lautet, die Weiterbildung für die in Rede stehende Ortsbezeichnung ebensowohl mit $\mathrm{n}$ wie mit 1 geschehen konnte, die Namen Magadan wie Magdala also durchaus gleichberechtigt und gleichwertig sind. An den Umstand, wie auch sonst am Wortschluß von Eigennamen 1 und $\mathrm{n}$ wechseln, sei nur erinnert (z. B. Jesreel jetzt Zer'în

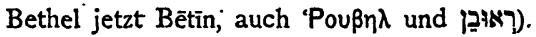

Nach dem allen wird man sich schwerlich dem Urteil verschließen können, da@ die Abschreiber, die Mt I5, 39 Magadan mit Magdala vertauschten, so ziemlich das Richtige trafen, gleichviel ob sie mit Überlegung handelten oder nicht.

I Eusebius' Onom. läßt in dieser Frage wie öfter völlig im Stich, da seine Notiz 2u Mayedaiv (134, 18. ed. Klosterm.) einfach sinnlos ist. 